\title{
Pneumothorax and Pneumomediastinum with Subcutaneous Emphysema Following Parathyroidectomy and Thyroidectomy
}

\author{
Russel Krawitz ${ }^{1}$, Anthony Glover ${ }^{2}$, Ahmad Aniss $^{3}$, Mark Sywak ${ }^{4}$, Leigh Delbridge ${ }^{5}$, Stan Sidhu ${ }^{6}$
}

\begin{abstract}
Background: Thyroidectomy and parathyroidectomy have become safe procedures with low postoperative morbidity and complication rates-hypocalcemia, RLN injury and postoperative hematoma being the most common. In our institution the risk of hematoma following sutureless technique is $1 \% .{ }^{1}$ Pneumothorax following thyroidectomy and parathyroidectomy has only been reported a few times in the literature without a clear etiology.

Materials and methods: Retrospective review of the complication database of the Royal North Shore Endocrine Surgical Unit from 2000 to 2018. Results: Three cases of pneumothorax or pneumomediastinum were found following thyroidectomy or parathyroidectomy with an incidence of $0.02 \%$. A recent case of pneumomediastinum and subcutaneous emphysema following an open parathyroidectomy was attributed to the Valsalva maneuver at the end of the case. Two further cases of pneumothorax at our institution occurred post parathyroidectomy. In both cases, a laryngeal mask was used and Valsalva maneuver (VM) was not performed. All cases were managed conservatively and made a full recovery. Conclusion: The combination of pneumomediastinum with subcutaneous emphysema in the most recent case is likely from a ruptured bulla secondary to Valsalva maneuver or lung injury during mediastinal dissection. This likely caused an air leak with gas tracking up into the neck from the mediastinum. The probable etiology in the other two cases is a negative mediastinal pressure created from laryngospasm with an open neck wound and dissection in the inferior neck and superior mediastinum.

Keywords: Parathyroid, Parathyroidectomy, Pneumomediastinum, Pneumothorax, Subcutaneous emphysema, Thyroidectomy, Valsalva maneuver. World Journal of Endocrine Surgery (2019): 10.5005/jp-journals-10002-1259
\end{abstract}

\section{INTRODUCTION}

Thyroidectomy and parathyroidectomy have become safe procedures with low postoperative morbidity and complication rates, hypocalcemia and RLN injury being the most common. Postoperative bleeding leading to cervical hematoma is an extremely serious complication of thyroidectomy with potential respiratory arrest from airway compromise. In our institution the risk of hematoma following sutureless technique is $1 \%$.

Many strategies are used to prevent postoperative hematoma with meticulous hemostasis at the end of the procedure being paramount. Valsalva maneuver (VM) is a common technique used prior to wound closure to detect occult bleeding and prevent postoperative hematoma.

Valsalva maneuver causes a number of complex physiological changes, but in the operative setting for neck surgery, the most important effect is the increased venous pressure within the jugular veins as a result of decreased venous return from the elevated intrathoracic pressure. ${ }^{2}$ By increasing venous pressure occult bleeding sites will become evident for the surgeon to then control by any number of surgical techniques.

Valsalva maneuver is generally a safe procedure but there are reports in the literature of complications resulting from barotrauma. These include neurological injury such as stroke, VM induced retinopathy, pneumothorax and pneumomediastinum as a result of alveolar or bullous rupture. ${ }^{2}$

A recent case of pneumomediastinum and subcutaneous emphysema following an open parathyroidectomy, was attributed to the VM at the end of the case, but this series will report two further cases of pneumothorax which occurred post parathyroidectomy both in which VM was not performed.
1-6University of Sydney Endocrine Surgical Unit, Royal North Shore Hospital, Northern Sydney Local Health District, Sydney, Australia

Corresponding Author: Russel Krawitz, University of Sydney Endocrine Surgical Unit, Royal North Shore Hospital, Northern Sydney Local Health District, Sydney, Australia, Phone: +61 447022315, e-mail: russelkrawitz@gmail.com

How to cite this article: Krawitz R, Glover A, et al. Pneumothorax and Pneumomediastinum with Subcutaneous Emphysema Following Parathyroidectomy and Thyroidectomy. World J Endoc Surg 2019;11(2):46-48.

Source of support: Nil

Conflict of interest: None

\section{Materials and Methods}

The study group included all patients having thyroidectomy or parathyroidectomy that were treated by the University of Sydney Endocrine Surgical Unit based at Royal North Shore Hospital, Sydney between 2000 and 2018. Data were stored in a dedicated endocrine surgical database and was audited on a monthly basis.

Included in this study were patients who had a pneumothorax or pneumomediastinum as a complication of thyroid or parathyroid surgery.

\section{Results}

Between 2000 and 2018 a total of 15,000 thyroidectomies and parathyroidectomies were performed. During this period, three patients had a recorded pneumothorax or pneumomediastinum as

(c) The Author(s). 2019Open Access This article is distributed under the terms of the Creative Commons Attribution 4.0 International License (https://creativecommons. org/licenses/by-nc/4.0/), which permits unrestricted use, distribution, and non-commercial reproduction in any medium, provided you give appropriate credit to the original author(s) and the source, provide a link to the Creative Commons license, and indicate if changes were made. The Creative Commons Public Domain Dedication waiver (http://creativecommons.org/publicdomain/zero/1.0/) applies to the data made available in this article, unless otherwise stated. 
a complication of surgery, giving this complication an incidence of $0.02 \%$. All three patients had parathyroidectomy and in one case a combined parathyroidectomy and thyroidectomy was performed.

All three patients were managed conservatively without need for an intercostal catheter and all made a full recovery. Details of the individual cases are presented.

\section{Patient 1}

A 66-year-old female with primary hyperparathyroidism and multinodular goiter who underwent a 4-gland exploration with right inferior parathyroidectomy and right hemithyroidectomy in early 2018 via traditional open approach (Fig. 1).

Her past medical history included hypertension and she was an active cigarette smoker. She had no previous neck surgery but had a laparoscopic hysterectomy 6 months prior.

At operation, a parathyroid adenoma was not easily identifiable and $660 \mathrm{mg}$ of fibrofatty tissue with a right inferior parathyroid adenoma embedded within was resected. After further exploration a left inferior parathyroid gland was not identified and paratracheal tissue on the left was resected along the course of the RLN and the left thymus was resected. A right hemithyroidectomy was then performed without difficulty. The right RLN and EBSLN were identified and preserved and function confirmed with a neuromonitor.

Prior to skin closure a VM was performed as per the surgeon's usual protocol of $40 \mathrm{~mm} \mathrm{Hg}$ for 30 seconds. Whilst normal saline was in the wound, an air leak was noted coming from behind the sternum arising from the anterior superior mediastinum, below the level of surgical dissection. The neck was closed in the usual fashion.

Within an hour post surgically the patient developed pain in the upper chest and neck and several hours later developed subcutaneous emphysema behind her wound.

Postoperative CXR identified a pneumomediastinum with surgical emphysema without obvious pneumothorax. She was monitored in the high dependency unit for 36 hours and was then returned to the ward. The subcutaneous emphysema resolved and she was discharged home without ongoing issue.

\section{Patient 2}

A 74-year-old female with primary hyperparathyroidism who underwent minimally invasive parathyroidectomy for a large

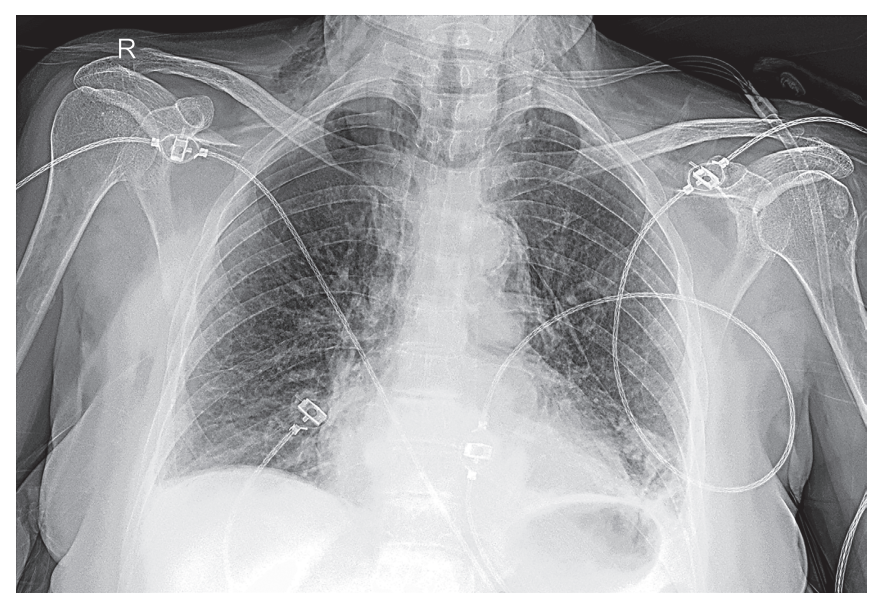

Fig. 1: Patient-1 postoperative chest X-ray showing pneumomediastinum with cervical subcutaneous emphysema descended right parathyroid adenoma found in the tracheaesophageal groove below the lower pole of the right thyroid lobe. After removal of the adenoma PTH levels fell appropriately. Postoperatively she experienced chest pain and was found to have an apical pneumothorax on CXR. This was managed conservatively and patient recovered fully.

A laryngeal mask was used during surgery and no VM was performed during the operation.

\section{Patient 3}

A 49-year-old female with primary hyperparathyroidism who initially underwent a minimally invasive parathyroidectomy which was converted to an open parathyroidectomy after large multilobulated descended right parathyroid adenoma was identified and was technically not able to be removed through a keyhole incision. An enlarged left superior gland was also identified and resected. Both inferior glands were unable to be located, presumably lying deep within the thymus. She tolerated the procedure without issue but post operatively developed significant chest pain with a chest X-ray showing bilateral apical pneumothoraxes which were managed conservatively and the patient was discharged home without any ongoing concern.

No VM was performed but intraoperatively the patient developed laryngeal spasm and the laryngeal mask was changed to an endotracheal tube.

\section{Discussion}

This case series highlights an unusual complication following parathyroidectomy, which has been reported previously on four patients following minimally invasive parathyoidectomy. ${ }^{3}$ The etiology for this is unclear and it was presumed that VM was responsible in the first case, but no VM was performed in the other two cases. The etiology in the most recent case is likely different to the previous two cases in this series and what has previously been reported in the literature, with a distinction that in the most recent case, the patient also developed subcutaneous emphysema (SE) in the neck which is likely driven by an ongoing air leak, as opposed to the earlier two cases which is more likely related to dissection in the upper mediastinum with negative intrathoracic pressures causing air to track downwards into the chest.

There are multiple etiologies associated with SE, normally as a result of puncture or rupture of part of the respiratory or gastrointestinal system with gas/air being trapped in the subcutaneous tissue. ${ }^{4}$ Soft tissue infections with gas forming bacteria are also a potential cause of SE. ${ }^{4}$ Cervical SE is most often seen following pneumothorax and with ICC insertion as well as with procedures of the cervical trachea and larynx but cervical SE has even been reported following a case of perforated colonic diverticula. ${ }^{5}$

SE has also been reported as a rare complication of thyroidectomy as a result of tracheal injury. Late SE has been associated with tracheal necrosis following thyroidectomy. ${ }^{6,7}$ Another case report describes a young patient with SE on day nine post thyroidectomy and at reoperation a tracheal tear without necrosis was identified. ${ }^{8}$

In the first case the combination of cervical SE and pneumomediastinum, with air bubbling during the VM makes a tracheal injury to be very unlikely as the leak was seen coming from the mediastinum. The two hypotheses that could have led to this combination of pathology is either, an apical bulla that ruptured from VM with a direct communication into the neck from upper 
mediastinal dissection causing injury to the pleura, or it could be due to direct injury of the pleura and apex of the lung itself. In this second theory, the VM would have only been diagnostic in alerting the surgeon that this injury would have occurred as opposed to being the causal factor.

Valsalva maneuver is generally considered a very safe maneuver, low and colleagues at the Mayo Clinic have performed 4,000 awake VM's a year since 1993 and have reported no complications. ${ }^{2}$ The literature though does report multiple cases of rare complications related to VM, with cases of Valsalva retinopathy, hyphema, pneumomediastinum and pneumothorax being some. ${ }^{2}$ If VM was the cause of the subcutaneous emphysema, this iatrogenic injury could have been avoided by not performing this technique.

It is also important to assess the efficacy of VM for hemostasis. A 2010 Cambridge study "Hemostasis in head and neck surgical procedures: Valsalva manoeuvre vs Trendelenburg tilt" compared these two techniques in detecting bleeding points to achieve adequate hemostasis. Trendelenburg tilt was defined as 30 degrees head down tilt and was carried out for a median of 6 minutes compared to VM which was carried out for a median of 30 seconds at $30 \mathrm{~cm}$ PEEP. A total of 50 patients were enrolled in this study and majority of operations were hemi/total thyroidectomy. "The Trendelenburg technique proved more sensitive in identifying bleeding vessels that were not seen by Valsalva (113/134 bleeding vessels identified). It is worth noting that, in five cases, significant bleeding that required stitching or tying was missed using VM." The authors concluded that Trendelenburg tilt was superior to VM and their institution now only performs 30 degree head down tilt without the use of VM. ${ }^{9}$

\section{Conclusion}

This paper reports a rare complication of parathyroidectomy. The initial concern prior to writing this paper was that VM was implicated in causing a pneumothorax. From this case series it is shown to be extremely uncommon with only one case in 15,000 cases in the last 20 years, in which a VM could be implicated. A number of hypotheses as to the causality of this rare complication have been presented in this paper, with the constant risk being cervical dissection of the upper mediastinum.

Valsalva maneuver is a common technique used at the end of head and neck surgical cases to identify bleeding sites. The rarity of this complication should not factor in to the decision made by the surgeon as to whether or not to use a VM at the end of the case for bleeding site identification.

Valsalva maneuver has utility in diagnosing this complication intraoperatively. Bubbling during VM can alert the astute surgeon of this complication and should trigger a management algorithm including use of a drain in the neck wound and post-operative CXR with the appropriate management of pneumothorax.

\section{References}

1. Chang L, Neill CO, et al. Sutureless total thyroidectomy: a safe and cost-effective alternative. ANZ J Surg 2011;81:510-514. DOI: 10.1111/j.1445-2197.2010.05492.x.

2. Kumar CM. Intraoperative Valsalva maneuver: a narrative review ratoire: une revue narrative Manœuvre de Valsalva perope. Can J Anesth 2018;65:578-585. DOI: 10.1007/s12630-018-1074-6.

3. Guerrero MA, Wray CJ, et al. Minimally Invasive Parathyroidectomy Complicated by Pneumothoraces: A Report of 4 Cases. J Surg Educ 2007;64(2):101-107. DOI: 10.1016/j.jsurg.2006.10.006.

4. Vilaça AF, Reis AM, et al. The anatomical compartments and their connections as demonstrated by ectopic air. Insights Imaging 2013;4(6):759-772. DOI: 10.1007/s13244-013-0278-0.

5. Fleischer BP, Carney MJ, et al. Perforated colonic diverticula presenting as periorbital and cervical subcutaneous emphysema. Am Surg 2017;83(6):677-678.

6. To EW, Tsang WM, et al. Tracheal necrosis and surgical emphysema: A rare complication of thyroidectomy. Ear Nose Throat J 2002;81(10): 738-741. DOI: 10.1177/014556130208101016.

7. Alevizos $L$, Tsamis $D$, et al. Delayed tracheal rupture after thyroidectomy. Am Surg 2012;78(4):E227-E228.

8. Mazeh H, Suwanabol PA, et al. Late manifestation of tracheal rupture after thyroidectomy: case report and literature review. Endocr Pract 2012;18(4):e73-e76. DOI: 10.4158/EP11344.CR.

9. Moumoulidis I, Pero MMD, et al. Haemostasis in head and neck surgical procedures: Valsalva manoeuvre vs Trendelenburg tilt. Ann R Coll Surg Engl 2010;92(4):292-294. DOI: 10.1308/003588410X1266 4192076412. 